\title{
CULTURA DIGITAL E INVESTIGACIÓN EN CIENCIAS SOCIALES: PRINCIPALES DESAFÍOS
}

\author{
Teresa Pacheco-Méndez \\ Universidad Nacional Autónoma de México
}

https://doi.org/10.33676/EMUI_nomads.54.09

\begin{abstract}
Resumen.- La presencia de las tecnologías de la información y la comunicación, así como la gama recursos digitales disponibles, plantea en todos los campos de conocimiento la necesidad de reformular sus habituales referentes y puntos de partida. Entre ellos, destacan: objetos, objetivos, procedimientos, y perspectivas que dan sentido y vigencia al conocimiento en cuanto a sus posibilidades técnicas, cognitivas y epistemológicas. En este trabajo, analizamos desde diferentes enfoques el comportamiento de tal desafío en el caso de la investigación social; de ahí que su contenido gire en torno a tres ejes de análisis: las Tic como herramientas de investigación, como un objeto más de estudio de las ciencias sociales, y como un componente indisociable de una realidad en permanente transformación que afecta a los modos de ver y de reflexionar sobre la actual constitución de los objetos de estudio de lo social.
\end{abstract}

Palabras clave: ciencias sociales, investigación, TIC, recurso técnico, objeto de estudio, uso social.

\section{Digital culture and social science research: main challenges}

\begin{abstract}
The emergence of information and communication technologies, as well as the range of available digital resources, raises the need in all areas of knowledge to reformulate their usual references and starting points. Among them, they highlight objects, objectives, procedures, and perspectives that give meaning and validity to knowledge in terms of its technical, cognitive and epistemological possibilities. In this work, we analyze from different approaches the behavior of such a challenge in the case of social research; for that reason the content of this paper turn around three axes of analysis: ICTs as research tools, as an object of study in the social sciences, and as an inseparable component of a reality in permanent transformation that affects the ways of seeing and to reflect on the current constitution of the objects of study of the social.
\end{abstract}

Keywords: social science, research, ICTs, technical resource, object of study, social use.

\section{Introducción}

En cada momento histórico las ciencias sociales han ido redefiniéndose, replanteándose y reconformándose como ámbito de pensamiento. Es así como, hoy en día, las identificamos como un campo de conocimiento amplio y diverso que posee una larga trayectoria histórica en la que han intervenido, tanto condiciones sociales, políticas y culturales diversas, como debates cognitivos de distinto origen; entre estos últimos destacan los interdisciplinarios, los filosóficos, y los epistemológicos.

Es esta experiencia sociohistórica -y la reflexividad desplegada en torno a este ámbito de pensamiento- lo que define las posibilidades de las ciencias sociales para enfrentar los actuales desafíos cognitivos y técnicos, frente al 
uso, aplicación y apropiación en la investigación de las nuevas tecnologías de la información y la comunicación (TIC). Sin duda, este nuevo ajuste de las ciencias sociales a actuales condiciones -en cuanto a sus orientaciones teóricas, objetos, métodos y categorías-, plantea a este ámbito de pensamiento nuevas necesidades para reestructurarse, y redefinir su área de influencia.

En este trabajo se abordan las ventajas y desventajas producidas por la introducción de las Tic en el campo de la investigación en ciencias sociales. Se distingue, por un lado, su uso y aplicación como herramienta, como modo expresivo, y como un objeto más de estudio. Por otro, se le visualiza como un componente intrínseco de la realidad social que obliga a poner en marcha nuevas operaciones epistemológicas de apropiación, que posibiliten el desarrollo de la capacidad reflexiva necesaria para comprender, con una perspectiva histórica, el fundamento de la acción individual y de la dinámica social actual.

\section{La naturaleza de los cambios}

En la historia de la sociedad, el componente tecnológico ha jugado un papel relevante en la profundidad y alcance de los cambios sociales. La introducción de avances tecnológicos ha propiciado en distintos momentos y coyunturas, modificaciones en lo simbólico y social de la actividad humana. Todo ello ha planteado nuevos enigmas de conocimiento, desempeñándose con frecuencia como eventuales potenciadores de la capacidad interpretativa del científico social: "la tecnología-quiérase o nomodifica las conductas sociales y condiciona de una $u$ otra manera a los individuos que la usan." (Ayala, 2011:49). Se transforman prácticas sustantivas en la investigación como son la lectura y escritura, introduciendo cambios cognitivos que abarcan desde el acceso a la información, su depuración, la generación y la puesta en circulación del conocimiento producido.

A diferencia del campo de las ciencias exactas y naturales, en la investigación social prevalece la tendencia a la extensa y vasta argumentación como rasgo distintivo en la construcción del discurso científico, "el conocimiento debe procesarse, para lo cual no se necesita velocidad, sino reflexión, y para que realmente el saber se convierta en tal, el individuo debe guardar la información en su memoria." (Ayala, 2011: 56) A ello se debe en parte que algunas disciplinas sociales como lo son la historia y la sociología manifiesten una peculiar inclinación por el recurso del texto impreso -documentos, libros y revistas- ya sea para la consulta, la indagación, o bien, para la producción y circulación de conocimiento. En estos campos disciplinarios aún no queda del todo establecido y reconocido, el acceso a fuentes y referencias provenientes de medios digitales, así como tampoco validada la pertinencia de la socialización del conocimiento producido por la investigación a través de estos medios. Para el caso de la historia, Pons (2013) es categórico: "En realidad, lo que observamos es una clara tensión entre el modelo tradicional y los nuevos 
soportes que no se resolverá mientras la disciplina no acierte a reconocer y a valorar los nuevos tipos de escritura y de difusión" (p. 271). Tensiones que, a juicio del autor, aumentan en uno y otro sentido, obligando a pensar en nuevas formas de leer y escribir, así como de producir y transmitir conocimiento.

La frecuencia con la que las nuevas tecnologías han sido consideradas en el campo de las humanidades y las ciencias sociales como un medio que ha transformado los modos de hacer, trabajar y difundir, no ha hecho más que plantear un debate entre posiciones divergentes $e$ insuficientemente abordadas. Para Pons (2013), tal debate considera a las nuevas tecnologías, por un lado, como una herramienta y como un medio expresivo, y por otro, como objeto propio de análisis. Como herramienta, las tecnologías acortan procesos y tiempos, permitiendo hacer muchas más tareas y facilitando otras; como medio de expresión, es en ellas donde prima la participación activa, condición que "implica explorar el potencial interactivo de la expresión mutimedia, en la que el texto deja de ser el vehículo fundamental". Por último, considerar a las nuevas tecnologías como un objeto más de estudio, plantea -según el autor- una problemática más compleja, ya que implica "introducir un objeto nuevo dentro de campos académicos de larga tradición" (p. 39)

Esta consideración del recurso tecnológico en la investigación social es también compartida por Bourdeloie (2013), quien afirma que: "la digitalización no es solamente una herramienta al servicio de la investigación sino también un objeto de investigación. Es al mismo tiempo instrumento, método, terreno y objeto de investigación" (p.19) ya que permite diseñar nuevos corpus (documentos impresos, sonoros, visuales, etc.), poniendo a disposición del investigador acervos de datos poco comparables con los antes ofrecidos por la etnografía tradicional; datos que hoy adquieren el estatus de Big data. (p.20)

Un mayor alcance sobre el impacto tecnológico en la investigación socíal es el planteado por San Segundo y Martínez (2012), quienes consideran que el efecto de las tecnologías es aún más considerable que los antes evocados, ya que logra incluso trastocar el referente cognitivo y epistemólógico del conocimiento:

...el cambio tecnológico que estamos experimentando hace impredecible el futuro, todas las tecnologías nos han modificado como especie, la tecnología de la información electrónica nos influye y modifica, para encontrar, almacenar y compartir información puede, literalmente, alterar nuestros procesos neuronales. Es posible que con las nuevas modalidades de conocimiento se lleguen a activar nuevos módulos o funciones de la mente; al mismo tiempo, funciones que hemos tenido activadas durante siglos, volverán a su estado de reposo. Este cambio está teniendo efectos profundos en el contenido de los conocimientos y en la forma en que están organizados. (p. 419) 
A tales consecuencias cognitivas se suma la afectación que las tecnologías tienen en la investigación social no solo sobre las formas de pensar sino incluso, sobre nuestras concepciones de sociedad, comunidad, grupo y relación interpersonal. "Imposible pensar que una trasformación tecnológica [...] no se encuentre acompañada de una transformación de los modos de pensar con la tecnología" (Souchier, 1996 cit. por Bourdeloie, 2013: 23). Sin olvidar el fundamento informático y matemático que abarca la digitalización, su USO, aplicación y apropiación en la investigación social no puede soslayar la relevancia del papel de la subjetividad de lo social.

Esta diversidad de perspectivas sobre la injerencia de los recursos tecnológicos en el pensamiento social marca, a grandes rasgos, los ángulos del debate que las Tic, y en particular la cultura digital plantean en el terreno de los procesos de producción de conocimiento de lo social. Un debate que deja al descubierto la necesidad de poner en marcha operaciones epistemológicas que, fundadas en un esfuerzo de comprensión crítica permita al individuo situar y administrar, desde el punto de vista cognitivo, el volumen y ritmo de crecimiento de la información puestos en circulación a través de los medios electrónicos. "Una operación que incluye habilidades críticas y cognitivas y operaciones semióticas -codificar, descodificar, interpretar y evaluar textos mediáticos-. Es esencialmente una tarea de aprender a saber en tanto que formación crítica del pensar." (Crespo, 2010: $s / p)$

\section{Potencial técnico, cognitivo y epistemológico de la cultura digital.}

La delimitación y especificidad de los objetos de estudio en las ciencias sociales se caracteriza por la inter-subjetividad constituyente que los delimita y les da significado; una inter-subjetividad donde se articulan diferentes niveles de concreción de una realidad social dinámica y en permanente transformación. Son unidades de estudio que se presentan no como objetos claramente definidos, sino como campos problemáticos a los que es necesario aproximarse en función de las potencialidades y modalidades de su desenvolvimiento temporal y de su dinámica constitutiva. Esta peculiaridad de las ciencias sociales permite establecer una distinción básica entre un hecho social entendido como un producto histórico acabado, o bien, como un campo problemático articulado producente de nuevas realidades (Zemelman, 1996).

Son estos rasgos, característicos de las ciencias sociales, los que hoy en día explican por qué "lo que era real, sólido, seguro, perdurable y nacional, se volvió virtual, flexible, ambiguo, frágil, líquido, evanescente y global" (Gatti, cit. por Peirone, 2013 p, 3). Un punto de crisis cuyo alcance socio-institucional ha dado lugar a

"la emergencia de un contexto socio-tecnológico que complejiza aún más el escenario introduciendo un nuevo patrón cultural... la cultura digital reformula los vínculos interpersonales y altera las prácticas políticas, 
comerciales, formativas e intersubjetivas. Nada se sustrae a la embestida, y desde la academia hasta la familia, pasando por la normativa jurídica y los modelos de producción, todos los actores sociales se ven compelidos a revisar sus roles y fundamentos" (Peirone, 2013: p. 3)

En medio de tal complejidad, la realidad sociohistórica difícilmente puede ceñirse a las delimitaciones claras y fijas exigidas por la digitalización y el uso de herramientas tecnológicas, ya que éstas no admiten la "ambigüedad... [precisamente] por la incapacidad de esa tecnología para manejar lo equívoco, lo incompleto o contradictorio" (Pons, 2013: 284). Es aquí donde se plantea la necesidad de distinguir los desafíos y posibilidades técnicos, cognitivos y epistemológicos a los que se enfrentan los fines y propósitos de la investigación social. Situarlos en su debida proporción en el contexto de la cultura digital, permitirá distinguir con mayor claridad, y desde una perspectiva sociohistórica, el fundamento de la acción individual y de la actual dinámica social.

\subsection{Ventajas y desventajas técnicas. Las tic como herramienta y como objeto de estudio.}

\section{Ventajas}

Entre las principales ventajas técnicas obtenidas a través del uso de los recursos tecnológicos en la investigación social -estudiadas con amplitud por Dwyer, 2004; Ayala, 2011; Escalante, 2016 y muchos otros)-, destaca el alcance y visibilidad de los procesos de generación del conocimiento, así como su impacto más allá de las tradicionales fronteras geopolíticas. Esto se refleja principalmente a través de mecanismos tales como:

- El contacto abierto a considerables acervos de la información y conocimiento, abarcando el desarrollo de una infraestructura y de aplicaciones para el acervo, acceso, y manejo de grandes bases de datos:

"el acceso casi ilimitado a la información y el intercambio de los conocimientos permiten la preservación de los saberes heredados, al tiempo que se crean nuevos saberes relacionados con la tecnología digital. Los saberes ya no son patrimonio de un grupo privilegiado, sino que se democratizan y se comparten mientras los hábitos de lectura se modifican debido a la influencia del hipertexto" (Ayala, 2011:58)

El más elemental empleo de un equipo de cómputo contribuye al desarrollo de la habilidad para manejarlo, y administrar a través de sus distintos buscadores, el volumen y variedad de información disponible en la red de redes: Internet. La versatilidad y acortamiento de los tiempos es considerable y en consecuencia, más eficiente la búsqueda, localización y depuración de información. La destreza digital adquirida es progresiva y relevante para su uso y aplicación en los distintos escenarios que se proyectan a futuro. Las formas de lectura, escritura, expresión verbal, sonora y corporal se diversifican y se transforman de manera constante y permanente. 
- La generación de nuevas condiciones y plataformas de comunicación, circulación, difusión y transferencia de conocimiento entre los distintos campos científicos y los distintos sectores de la sociedad. En el terreno específico de la difusión del conocimiento, los mecanismos de circulación de la información estimulan el crecimiento de una oferta editorial que permite canalizar, socializar $y$, sobre todo, poner en el terreno del debate internacional los productos de la investigación en lapsos considerablemente más cortos que los ofrecidos por los tradicionales medios editoriales impresos.

- El establecimiento de redes sociales para fortalecer el trabajo colaborativo, lo que redunda en una importante contribución para la formación de movimientos cuyo principal soporte es la actual estructura comunicativa (Internet, telefonía móvil, etc.)

\section{Desventajas}

- Entre las principales desventajas técnicas figura el acceso condicionado a los recursos tecnológicos indispensables para el acceso a la información y la comunicación; dificultades que por lo general están sujetas a las condiciones socioeconómicas de cada sector social, y al diferenciado desarrollo de los países. Sobre el tema, Castells (2001) asegura que existe

"una gran diferencia de conectividad y observamos que aquellas personas que no tienen acceso a Internet tienen una debilidad cada vez más considerable en el mercado de trabajo. Observamos también que los territorios no conectados a Internet pierden competitividad económica internacional $y$, por consiguiente, son bolsas crecientes de pobreza incapaces de sumarse al nuevo modelo de desarrollo." (s/p)

- El contenido e información que circula a través de medios digitales es de muy diversa manufactura, oscilando desde aquel material vinculado con el quehacer científico, hasta el asociado con la opinión común. A tal contraste y diversidad de información, se suma la disparidad de condiciones y capacidades educativas y culturales existentes para poder optimizar el uso de la oferta tecnológica -donde la Internet ocupa un lugar relevante. Con ello se hace referencia a una "capacidad de aprender a aprender, esa capacidad de saber qué hacer con lo que se aprende, esa capacidad es socialmente desigual y está ligada al origen social, al origen familiar, al nivel cultural, al nivel de educación." (Castells, 2001: s/p)

- Por la relación entre su naturaleza técnica, y la complejidad sociocultural que caracteriza a la diversidad de tipos de usuario, las Tic no siempre dan lugar a la modificación de prácticas de uso ya sea en un sentido transformador e innovador, o bien, en uno más bien reificador y reproductor de modelos estandarizados. La gama de respuestas entre teles extremos se amplifica de acuerdo con el contexto de referencia.

"usuarios, tecnologías e instituciones tienen variadas interrelaciones que influyen de manera considerable en el desarrollo de las TIC.... existen disímiles 
tipos de usuarios que incorporan en sus prácticas cotidianas diversos usos y con ello variadas formas de acuerdo a los contextos sociales en que éstas se insertan". (Rueda, 2016:76)

Tanto ventajas como desventajas, la distinción que plantea el uso de las Tic en la investigación social es precisamente el tipo de operaciones que cada individuo despliega en el uso de tales recursos tecnológicos. Como lo analiza De Certau (2000, p.37) para el campo de la comunicación, lo relevante ya no es cuantificar tendencias, disponibilidad y distribución de recursos tecnológicos, sino conocer el repertorio con el cual los usuarios realizan operaciones que les son propias. Esto hace referencia a lo que, por un lado, la literatura francófona sistematizada por Serge Proulx y Josiane Jouët, define como los usos sociales de la técnica; y por otro, a los enfoques socioculturales de la apropiación en los procesos de comunicación, impulsados por Michel De Certau, Pierre Bourdieu, Delia B. Crovi, MartinBarbero, entre otros.

\subsection{Ventajas y desventajas cognitivas y epistemológicas. La tecnología como componente intrínseco de la realidad social.}

Más allá de considerarlas como herramienta y como objeto de estudio, las posibilidades ofrecidas por las Tic a la investigación social se circunscriben al terreno de lo que Proulx (2005) define como construcción social del uso de la tecnología; es decir, la observación e identificación de lo que los individuos efectivamente hacen con los objetos y dispositivos tecnológicos. Desde la perspectiva de una etnografía crítica, el autor asegura que es la recuperación de argumentos y evidencias proporcionadas por los actores de la investigación con respecto a las Tic-sin reducirlas ni descalificarlas- Io que posibilita la construcción subjetiva del sentido, distinguiendo sus respectivos contextos de uso, normativos, de relaciones sociales y de poder. (p. 8)

La particularidad y distinción en la noción de uso social cobra sentido y alcance en la consideración efectuada por De Certau (2000) al atribuirle al sujeto la capacidad táctica, es decir, la posibilidad autónoma de utilizar de manera cotidiana, vigilante y en el tiempo, las fallas del poder y de la estrategia delineada por las instituciones. "la táctica se encuentra determinada por la ausencia de poder, como la estrategia se encuentra organizada por el principio de un poder". La potencia, creatividad y autonomía del sujeto quedan del todo expuestas en la medida que "Mientras más débiles son las fuerzas sometidas a la dirección estratégica, más capaz será ésta de astucias". (pp. 40-45)

En el sentido planteado por De Certau, el uso social de las Tic tiende a multiplicarse y diversificarse en función del despliegue táctico efectuado por los individuos, frente a las estrategias delimitadas por las instituciones con respecto a la disponibilidad y distribución de medios y artefactos tecnológicos de comunicación e informáticos. Las posibilidades de 
individualización, autonomía y libertad de los individuos frente a las Tic son concomitantes con el alcance de las operaciones cognitivas y epistemológicas por ellos efectuadas en su vida social y cultural diaria.

El uso social y cultural de las tecnologías se inscribe en la variedad y pluralidad de procesos de construcción de identidades, lo que distintos autores de la sociología contemporánea definen como proceso de individualización, contrario al sentido del individualismo institucionalizado. Es el caso de Beck (2001) para quien, en un contexto de una modernidad avanzada, la

"Individualización significa, en primer lugar, el proceso de desvinculación y, en segundo lugar, el proceso de revinculación a nuevas formas de vida de la sociedad industrial en sustitución de las antiguas, en las que los individuos deben producir, representar y combinar por sí mismos sus propias biografías". (p. 28)

La individualización es considerada por el autor como "un espacio creativo para la renovación de la sociedad...En la modernidad desarrollada...la mutualidad y la comunidad humana ya no descansan en tradiciones sólidamente establecidas, sino...más bien en una colectividad paradójica de individualización recíproca" (Beck y Beck-Gernsheim, 2003 p. 30).

Estas reconsideraciones conceptuales rescatan no sólo el papel del sujeto social, sino también la intersubjetividad y su potencial para impulsar cambios en distintas direcciones. Colocan a la acción social en el centro de la escena, liberándola de los muros institucionales y de concepciones cerradas de cultura y tradición. "El sujeto de hoy, que se relaciona más con instituciones fragmentadas, ha pasado de la posición de reflexión a la de ser reflexivo" (Lash, en Beck y Beck-Gernsheim, 2003:13).

\section{Ventajas}

- En lo cognitivo.

Desde el punto de vista cognitivo, el debate sobre el papel de las Tic en la sociedad se plantea en dos direcciones. Por un lado, posiciones que defienden que "la exposición diaria a la alta tecnología -como computadoras, teléfonos inteligentes, videojuegos o buscadores- modifica los caminos neuronales y la activación de los neurotransmisores, con lo que gradualmente se afianzan en el cerebro nuevos caminos, mientras los antiguos se desdibujan." (Small y Vorgan, 2009 cit. por Ayala, 201 1: 56). Por otro lado, los que aseguran que "no es Internet lo que cambia el comportamiento, sino que es el comportamiento el que cambia Internet" (Castells, 2001: s/p)

En lo que sí es posible coincidir es en el hecho de que la tecnología actual, estimula nuevas formas de: comunicación producción, circulación, y acceso al conocimiento. "Internet no es sólo un difusor de viejos saberes, de libros ya escritos, sino un nuevo modo de escribir y de producir saber". (Martín 
Barbero, 2003, 28). La Internet se ha convertido en el "corazón de articulación de los distintos medios, de los multimedia...hay una capacidad de comunicación masiva no mediatizada por los medios de comunicación de masas" (Castells, 2001: s/p)

Los recursos digitales vienen a trastocar la base tradicional de los procesos de aprendizaje, proporcionando al individuo "posibilidades de ver, escuchar y seguir representaciones variadas de los datos, asegurando una actividad perceptiva antes inaccesible a los sentidos humanos en una situación formal" (Fagundes, 2007, s/p). De este modo, el entorno de aprendizaje se ve

"enriquecido con las herramientas digitales conversacionales posibilita representar patrones de significados y acciones, reflexionar sobre las propias representaciones, permitir la gradual asimilación de los propios recursos digitales con el foco no más en tareas impuestas desde la autoridad externa pero en los procesos subjetivos en interacción con los procesos objetivos sustentando los progresos en los usos del lenguaje personal" (Fagundes, 2007, $s / p)$.

Lo que es imposible soslayar, aún desde la perspectiva cognitiva, es la presencia de toda implicación y preocupación de naturaleza epistemológica, sociológica y sociocultural de la tecnología en la generación, uso, aplicación y apropiación de conocimiento. Esto significa que, así como el lenguaje, la tecnología es constitutiva del sujeto social y de su actividad; de ahí que sea indispensable en ciencias sociales, la configuración de un encuadre teórico y metodológico capaz de analizar el acoplamiento entre tecnología y prácticas sociales. "El objeto técnico es ante todo pensado como un hecho de cultura inseparable de las prácticas sociales que lo producen". (Albero y Thibault, 2009: p.57 y 59)

\section{- En lo epistemológico}

La primera consideración es apuntada por San Segundo y Martínez (2012), para quienes la episteme ha sido el lugar donde se posiciona un grupo humano para asignar significado a las cosas y al conocimiento científico. Es el conjunto de conocimientos que ha condicionado la comprensión e interpretación del mundo. (pp. 415-416) En estos términos, los autores aseguran que hoy en día, ya no es la episteme sino el pensamiento digital el que conforma nuestra realidad y su forma organizativa. "La teoría general de la organización del conocimiento que ha estado mediada por la oralidad y por la escritura, ahora lo va a estar por la información digital." (p. 419) Este argumento es más claramente expuesto de la siguiente manera: "El lenguaje estableció un orden de las cosas, las epistemes establecieron un orden de los saberes, y los soportes de la información electrónica van a establecer el orden de lo real. Ya que se ha conformado lo virtual no como representación sino como lo real" (p. 420)

Lo formulado por los autores pone el acento, para el caso de las ciencias sociales y la investigación, en la idea del objeto de estudio como una 
entidad en cuya dinámica y complejidad actuales, lo virtual es ahora parte constitutiva. Se trata de objetos de estudio situados en entornos que trascienden: el lenguaje formal de la ciencia, los consensos establecidos por las comunidades científicas, y las habituales las prácticas sociales y culturales de interacción, intercambio y comunicación.

En ciencias sociales, lo que antes representó la hibridación ${ }^{1}$ del conocimiento, ahora lo percibimos como un movimiento de diseminación tanto "de las fronteras entre las disciplinas del saber académico como entre ese saber y los otros, que ni proceden de la academia ni se imparten en ella de manera exclusiva... [son los] que provienen de la experiencia social y de las memorias colectivas." (Martín-Barbero, 2003: 20)

En tales condiciones de complejidad, la cultura digital interpela a la epistemología de las ciencias sociales, cuestionado su estructura como conjunto establecido de disciplinas formales (Bourdeloie, p. 10). Aunque todos los contenidos de la web se encuentren en un mismo plano de acceso, todos ellos están diferenciados no precisamente por alguna disciplina en particular, sino más bien por la intervención de quien los selecciona, los orienta, los articula, y les da una disposición jerárquica siempre temporal y provisional. La web no se reduce a contenidos e información de diversa manufactura, sino que representa también una plataforma novedosa de pensamiento que posibilita la formación de una infinita variedad de puntos de partida para el razonamiento. "...cada uno de nosotros debe reconstruir las totalidades parciales a su manera, siguiendo sus propios criterios de pertinencia, ...estas zonas de significación propias deberán ser móviles, cambiantes, en desarrollo (Levy, 1999:6)

Para la investigación social se trata, por un lado, de

"la des-jeraquización de los conocimientos para que sea posible dar cuenta de las dinámicas y transformaciones sociales, y, [por]...otro, la asunción de la pluralidad de los discursos y lenguajes sociales - desde la abstracción científica a la ficción literaria, pasando por la crónica y el relato de vida, el lenguaje escrito, el audiovidual y el hipertextual-, como diversos modos de acceso a y de expresión de la irreductible multidimensionalidad de lo social." (Martín-Barberó, 2003: 27)

La investigación social se posiciona frente a una sociedad, constituida por procesos, intereses, valores e instituciones, fundada en bases estructurales que se han visto veloz y profundamente transformadas. "no son sólo las fronteras entre los saberes las que se quedaron obsoletas, sino entre saberes y deberes, entre investigación y proyecto de sociedad." (Martín-Barberó, 2003: 29) En esta sociedad, la Internet "constituye en realidad la base material de nuestras vidas y de nuestras formas de relación, de trabajo y de comunicación. Lo que hace Internet es procesar la virtualidad y

\footnotetext{
1 Dogan y Phare (1993) lo definen así: el proceso donde se "...combinan los conocimientos especializados de diferentes dominios constituye lo que nosotros denominamos hibridación. Un campo híbrido es el resultado de la recuperación de zonas marginales de dos o varias disciplinas." (pp. 67-68 y ss)
} 
transformarla en nuestra realidad, constituyendo la sociedad red, que es la sociedad en que vivimos" (Castells, 2001: s/p)

A la particularidad de la virtualidad a la que se enfrenta el quehacer de las ciencias sociales se suma la condición de descentramiento y deslocalización del conocimiento. En términos de Martín-Barbero (2003), hoy en día, este conocimiento escapa de los lugares que legítimamente antes lo contenían, así como de las figuras sociales que lo detentaban y administraban (p.18); es el caso -por ejemplo, en la educación- del salón de clase, la biblioteca, los compartimentos disciplinarios, tos perfiles laborales, etcétera. Surgen así para el autor dos cambios. El primero es el descentramiento, es decir cuando el saber sale de los libros y del sistema educativo escolarizado e institucionalizado, y sale además del que ha sido su eje en los últimos cinco siglos: el libro. Esto no supone que el texto electrónico reemplace al libro, sino que viene a "des-centrar la cultura occidental impuesta no sólo a la escritura y a la lectura, sino al modelo entero del aprendizaje por linealidad y secuencialidad" (p. 19)

El segundo cambio es lo que Martín-Barberó denomina la deslocalización/destemporalización, es decir, cuando los saberes escapan de los lugares y de los tiempos legitimados socialmente para la distribución y el aprendizaje del saber (el programa de estudio, el año lectivo, el grado escolar, el grado académico alcanzado, entre otros); "la des-localización implica la diseminación del conocimiento, es decir, el emborronamiento de las fronteras que lo separaban del saber común" (p.20). Es también el caso del parámetro de la edad pertinente, como referente de aprendizaje de contenidos específicos, y que en la actualidad muta para constituirse en un saber y un aprendizaje continuo, como una experiencia a lo largo de toda la vida; un "convivir con un montón de saberes-sin-lugar-propio" (pp. 19-20)

Además de estos fenómenos sociológicos de descentramiento y deslocalización en el saber, los alcances de la virtualidad y de la digitalización, han reconfigurado la estructura de funcionamiento de la sociedad y de los movimientos sociales:

"hay un salto de los movimientos sociales organizados a los movimientos sociales en red en la base de coaliciones que se constituyen en torno a valores y proyectos... La transmisión instantánea de ideas en un marco muy amplio permite la coalición y la agregación en torno a valores" (Castells, 2001. s/p)

La transformación que internet ha provocado en la sociedad ha tenido mayores alcances, y al erigirse como "instrumento de participación ciudadana extraordinario, podría ser un instrumento de información de la clase política, de los gobiernos y de los partidos a los ciudadanos en su conjunto y de relación interactiva. (Castells, 2001: s/p) Los beneficios sociales que ello conlleve dependerán, no obstante, de la capacidad de aprendizaje y de transformación por parte de individuos, de la diversidad de sociedades y de sus respectivas condiciones socioculturales. 


\section{Desventajas cognitivas y epistemológicas}

El principal obstáculo al que se enfrenta la innovación en el conocimiento a través de los recursos tecnológicos es precisamente pensar que los cambios sociales son provocados por el desarrollo tecnológico y no a la inversa.

"frente a los que plantean que el cambio social viene desencadenado casi exclusivamente por el cambio tecnológico, hay que afirmar, apoyados en la historia, que han sido los cambios sociales y culturales los que han posibilitado la absorción de la oferta tecnológica y su desarrollo, siendo el cambio social posterior la consecuencia de un cambio previo" (Bouza, 2002: s.p.).

El contacto con las tic si bien ha abierto las posibilidades de un cambio cultural, sú apropiación no necesariamente supone una ruptura con los anteriores patrones de razonamiento; mientras para algunos el contacto con las Tic se ha traducido en un esfuerzo, tanto por hacerse de una mayor cantidad y diversidad de información, como por establecer puntos de articulación -aunque no siempre de coherencia- y de asociación entre los diversos contenidos disponibles, en otros casos los resultados sólo han reafirmado un uso exteriorizado de los medios y aparatos tecnológicos, sin que ello se traduzca en una experiencia cognitiva que apunte a una producción innovadora de contenidos de conocimiento.

Tal como lo afirman Darío y Bouza, la presencia de la tecnología en la vida diaria no da necesariamente como resultado un cambio cultural con respecto a su supuesta apropiación. Para Darío (2014) el uso de artefactos tecnológicos, la capacidad de resignificarlos y de modificar su uso y alcance, está sujeto no sólo a las posibilidades y a las limitaciones en cuanto a la voluntad para transformarlo, sino también a las concepciones de quienes se los apropian, los evitan, o los que incluso los rechazan. Ejemplo de ello es el uso que de ellos hacen las denominadas "minorías sociales o políticas, pequeñas comunidades, etnias o grupos juveniles a lo largo de todo el continente" (p. 25)

Bouza (2002) va más allá afirmando que tanto en sociedades desarrolladas como en las no desarrolladas, la introducción de recursos tecnológicos puede llegar a convertirse en un obstáculo a la innovación; evitarlo supone contar con la suficiente capacidad para impulsar los correspondientes cambios de mentalidad, formas de aprendizaje, y renovados parámetros de competencia; cambios sociales que, en estos casos, son los que determinarán una consecuente demanda tecnológica.

La capacidad de individuos y grupos para hacer frente a la resistencia al cambio social e institucional, antes que al cambio tecnológico es una disposición que transita en distintas direcciones, no siempre libre de obstáculos. a) La posibilidad de superar las fronteras institucionales materiales y del conocimiento formal, impide que razonamiento e ideas se flexibilicen y rearticulen de acuerdo con las circunstancias del presente. b) El potencial 
de las Tic y su aplicación extensiva a la problemática social, cultural y económica, tiende a verse restringida a los márgenes de acción establecidos por intereses de poder y control social. c) El alcance y el ritmo de la circulación, comunicación y uniformización de la información impulsados por las Tic, contrarrestan el poder de los individuos y de la sociedad para "procesar la virtualidad y transformarla en nuestra realidad" (Castells, 2001: s/p)

Asegurar el acceso a la información, mantener un permanente contacto con los contenidos en línea, y contar con el uso intensivo de los dispositivos de comunicación propios de la cultura digital, no ofrecen de manera mecánica garantía alguna de transformación en las estructuras de pensamiento. Esto se debe a que en ocasiones tales modificaciones redundan más en cambios de tipo ontológico sobre el conocimiento adquirido, que en rupturas epistemológicas generadoras de un conocimiento innovador. Una circunstancia que se asemeja a lo identificado por Pierre Bourdieu (cit. por Martín-Barbreró, 2003) como capital cultural; es decir,

"ese capital simbólico que emerge de la trayectoria de vida, el que va a ir configurando el hábitus, ese sistema de disposiciones durables, que, a través de experiencias y memorias, va a posibilitar o a obstaculizar la creatividad, la capacidad de innovación de los sujetos" (p. 25)

Para las ciencias sociales, el desafío se presenta desde la definición de una realidad y de unos objetos de estudio que, al estar mediados por las Tic, reconforman las formas y la dinámica de la interacción social. "Internet es apta para desarrollar lazos débiles, ...pero no es apta para crear lazos fuertes...la tendencia que se está desarrollando es hacia la disminución de la sociabilidad de base comunitaria física tradicional" (Castells, 2001: s/p). Esto lleva consigo los dilemas planteados por la deslocalización/destemporalización ya señalados por Martín-Barberó.

\section{Reflexión final}

En ciencias sociales, la cultura digital y los recursos tecnológicos no pueden permanecer sólo al servicio de las promesas técnicas ni de los cambios meramente cognitivos, deben más bien aspirar a crear nuevas formas de pensar lo social y lo humano acordes con la realidad actual y su movimiento. Como lo señala Peirone (2013), este cambio plantea a las ciencias sociales la siguiente consideración:

"somos seres anfibios que entran y salen permanentemente de dos mundos. Un mundo sostenido por convenciones, prácticas inerciales y presupuestos conceptuales que remiten a modelos de interpretación todavía dominantes, 
pero en default. Y otro emergente, urgido por la necesidad de objetivar e institucionalizar una alternativa que hasta ahora sólo tiene como referencia lo que ya no quiere y se ha vuelto ineluctablemente disfuncional" (p. 7).

La misión de las ciencias sociales tendrá que contribuir a la asimilación de estos dos mundos, al esclarecimiento de sus puntos de contradicción, y a situar de manera permanente la experiencia social -y no la opinión y el sentido común- en el centro de la empresa científica. Sólo por este camino, la cultura digital y los recursos tecnológicos podrán estar al servicio de la investigación social, de la sociedad y del conocimiento.

\section{Referencias Bibliográficas}

Albero, B. y Thibault, F. (2009). La recherche française en sciences humaines et sociales sur les technologies en éducation. Revue française de pédagogie (169) Descargado de http://rfp.revues.org/1434

Ayala-Pérez, T. (2011). Saber y Cultura en la Era Digital. Revista Austral de Ciencias Sociales (20) 41-59. Descargado de http://mingaonline.vach.cl/pdf/racs/n20/art03.pdf

Beck, U. y Beck-Gernsheim, E. (2003). La individualización: el individualismo institucionalizado y sus consecuencias sociales y políticas. Barcelona: Ediciones Paidós Ibérica.

Beck, U., Giddens, A. y Lash, S. (2001). Modernización reflexiva Política, tradición y estética en el orden social moderno. Madrid: Alianza editorial.

Bourdeloie, H. (2013). Ce que le numérique fait aux sciences humaines et sociales. Epistémologie, méthodes et outils en question. tic\&société, 7 (2). Descargado de https://ticetsociete.revues.org//1500

Bourdieu, R. (1995). 2. Pensar en términos relacionales. En Respuestas. Por una antropología reflexiva (pp. 167-175). México: Ed. Grijalbo.

Bouza, F. (2002). Innovación tecnológica y cambio social. En Las encrucijadas del cambio social (pp. 85-97). Madrid, Centro de Investigaciones Sociológicas: Descargado de http://pendientedemigracion.ucm.es/info/socvi/BOUZA/NUEVAl/Textos/inno va.pdf

Castells, M. (2001). Internet y la sociedad red. Ponencia. Descargado de http://www.uoc.edu/web/cat/articles/castells/print.html

Crespo-Fajardo (2010). Bases epistemológicas de la sociedad del conocimiento. Descargado de https://lluiscrespo.wordpress.com/2010/02/18/bases-epistemologicas-de-lasociedad-del-conocimiento/ 
Darío-Andrés, G. (2014). Una aproximación conceptual a la "apropiación social" de tic. Questión. Revista especializada en Periodismo y comunicación. (1) 43.4 Descargado de http://ri.conicet.gov.ar/bitstream/handle/11336/10767/CONICET Digital Nro.1 4350.pdf? sequence $=1$ \&isAllowed $=y$

De-Certau, M. (2000). La invención de lo cotidiano. El arte de hacer. México: Universidad Iberoamericana. Instituto tecnológico y de Estudios Superiores de Occidente.

Dogan, M. y Phare, R. (1993). Las nuevas ciencias sociales. México: Ed. Grijalbo.

Dwyer T. (2004). Tecnologías de información y comunicación. Sus impactos sobre la pedagogía, la investigación y los paradigmas en las ciencias sociales. Investigaciones sociales, 8 (12) 325-335. Descargado de http://sisbib.unmsm.edu.pe/bibvirtualdata/publicaciones/inv_sociales/N12_2 004/a 1.5.pdf

Escalante, Gómez Eduardo (2016). Nuevas rutas de trabajo académico mediante el uso herramientas digitales. Revista Argentina de Educación Superior. 8 (12). pp. 53-65.

Fagundes, L. (2007) La Escuela en la Cultura Digital: ¿̇una nueva inteligencia? Ponencia presentada en la sección Aprendizaje con las TIC. XXII Semana Monográfica Fundación Santillana de la Educación. "Las tecnologías de la información y la comunicación (TIC) en la educación: retos y posibilidades". (Madrid, 22 de noviembre de 2007). Descargado de http://www.oei.es/tic/santillana/cruz.pdf

Lévy, P. (1999). Cibercultura y educación. Disponible en http://cmapspublic2.inmc.us/rid=1142254294250_1146738664_6112/Cibercultu ra_y_educacion.pdf

Martín-Barbero, J. (2003). Saberes hoy: diseminaciones, competencias y transversalidades. Revista Iberoamericana de Educación (32) pp. 17-34 Descargado de http://rieoei.org/rie32a01.htm

Proulx, S. (2005). Penser les usages des technologies de l'information et de la communication aujourd'hui : enjeux - modèles - tendances. En, Vieira, Lise y Pinède, Nathalie éds, Enjeux et usages des TIC: aspects sociaux et culturels. (p. 7-20) Tome 1. Bordeaux: Presses universitaires de Bordeaux.

Pons, A. (2013) El desorden digital: Guía para historiadores y humanistas. Madrid: Siglo XXI de España.

Peirone F. (2013) La cultura digital, las nuevas prácticas sociales y los procesos de subjetivación. El abordaje de las ciencias sociales. Descargado de http://www.academia.edu/4125182/La cultura digital las nuevas pr\%C3\%A lcticas sociales y los procesos de subjetivaci\%C3\%B3n. El abordaje de las ciencias_sociales 
Rueda-Barrios O. B. (2016). Alfabetización digital y apropiación social: dimensiones que aportan en la construcción de ciudadanía. Puente Revista $\begin{array}{lllll}\text { Científica } 10 & \text { (1). pp. 73-77. Descargado de }\end{array}$ https://revistas.upb.edu.co/index.php/puente/article/view/7020 14 agosto 2017

San-Segundo-Manuel, R. y Martínez-Ávila D. (2012). El orden de los saberes y la organización digital. 20 Años del Capítulo Español de ISKO. Actas del $X$ Congreso ISKO Capítulo Español (Ferrol, 2011) pp. 413-421. Universidade da Coruña, España. Descargado de http://www.iskoiberico.org/wpcontent/uploads/2014/09/413-421_San-Segundo.pdf

Zemelman, H. (1996). Ideas metodológicas para el estudio de los sujetos sociales. En Problemas Antropológicos y utópicos del conocimiento (pp. 91130). México, El Colegio de México. Col. Jornadas. No. 126.

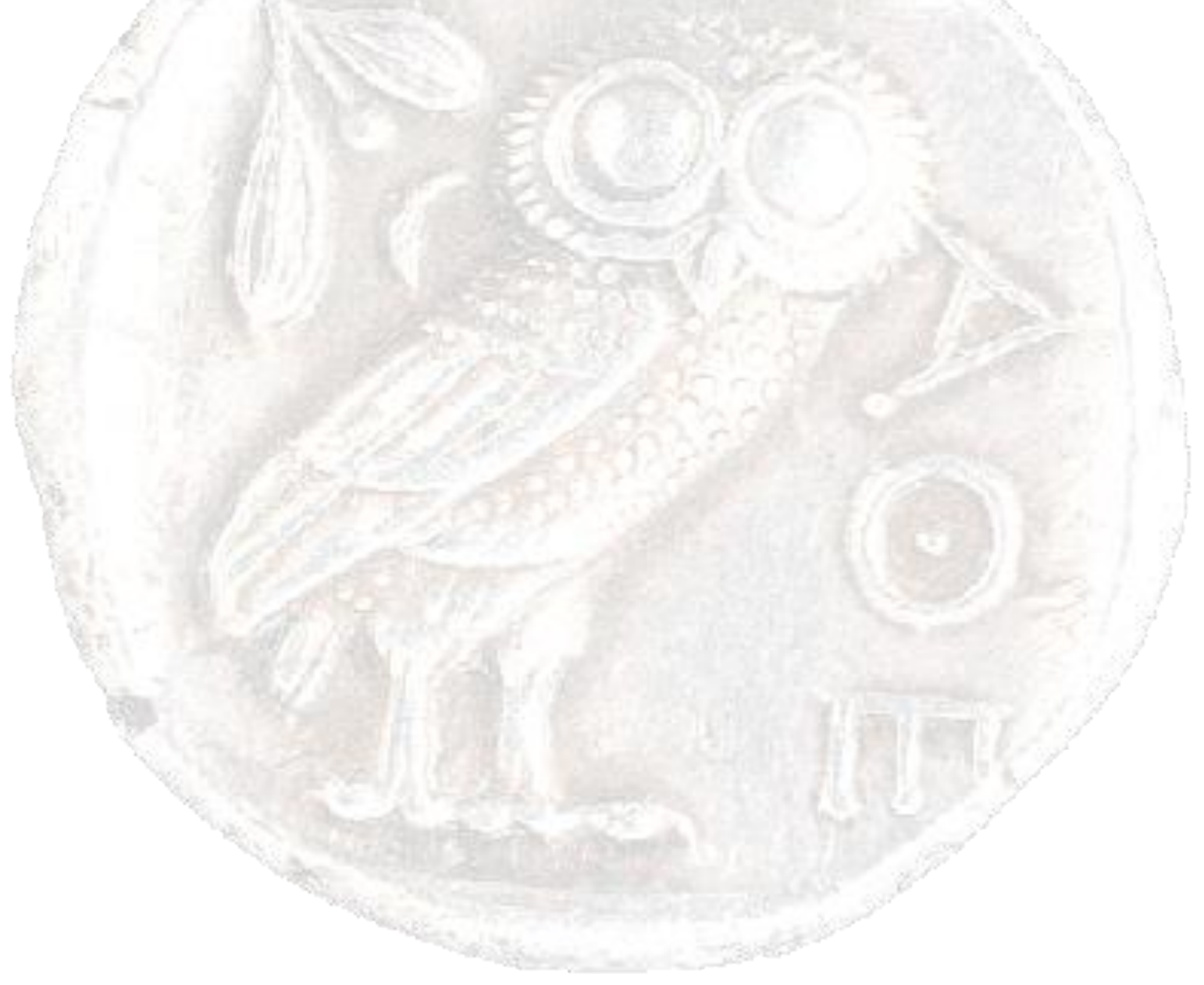

